\title{
Covid-19 em Pacientes Oncológicos: uma Revisão do Perfil Clínico-Epidemiológico
}

doi: https://doi.org/10.32635/2176-9745.RBC.2020v66nTemaAtual.1046

\author{
COVID-19 in Oncologic Patients: a Clinical-Epidemiological Profile Review \\ Covid-19 en Pacientes con Cáncer: una Revisión del Perfil Clínico-Epidemiológico
}

Rafaela Campos Alcântara'; Luiz Carlos Francelino Silva Junior²; Gabriel Monteiro Arnozo ${ }^{3}$; Tatiana Farias de Oliveira ${ }^{4}$; Fernanda Mayara Santos Santana5; Etvaldo Rodrigues da Silva Filho ${ }^{6}$; Aisla Graciele Galdino dos Santos ${ }^{7}$; Euclides José Oliveira da Cunha ${ }^{8}$; Saulo Henrique Salgueiro de Aquino ${ }^{9}$; Rodrigo da Rosa Mesquita ${ }^{10}$; Carlos Dornels Freire de Souza ${ }^{11}$

\section{Resumo}

Introdução: Desde o início da pandemia de Covid-19, estudos apontam que pacientes oncológicos representam um dos principais grupos de risco. Objetivo: Sintetizar a literatura no que se refere ao perfil clínico-epidemiológico de pacientes oncológicos com Covid-19. Método: Revisão integrativa da literatura a partir da base PubMed, utilizando os descritores COVID-19, SARS-CoV-2, 2019-nCoV, n-CoV e coronavirus combinados com cancer, tumor e malignancy. Resultados: Dos 821 artigos encontrados, nove textos foram incluídos na análise qualitativa, totalizando 39 indivíduos, com predomínio do sexo masculino ( $74,4 \%$ homens): China ( 6 textos, 35 pacientes), Nigéria (1 texto, 1 paciente), França (1 texto, 2 pacientes) e Dinamarca ( 1 texto, 1 paciente). Os cânceres de pulmão $(51,3 \% ; n=20)$ e de mama $(10,3 \% ; n=4)$ foram os mais prevalentes. Em 87,2\% (n=34), não foram relatadas informações sobre hábitos de vida. Os sintomas respiratórios $(46,8 \% ; \mathrm{n}=18)$ e febre $(43,6 \% ; \mathrm{n}=17)$ se destacaram. Seis pacientes foram a óbito $(15,4 \%)$, quatro receberam alta por cura $(10,2 \%)$, dois permaneceram internados até a publicação do estudo $(5,2 \%)$ e sobre os demais $(69,3 \%$, n=27) o manuscrito não informava o desfecho. Conclusáo: $\mathrm{O}$ cuidado adequado a esse grupo especial de pacientes quando infectados pelo novo coronavírus é fundamental em busca de melhores desfechos. Mais estudos ainda são necessários para uma melhor compreensão de como a Covid-19 se comporta em pacientes com câncer.

Palavras-chave: Infecçóes por Coronavirus; Neoplasias/epidemiologia; Revisão.

\section{Abstract}

Introduction: Since the beginning of COVID-19 pandemic, studies indicate that oncologic patients represent one of the main risk groups. Objective: To synthesize the literature addressing clinical and epidemiological profile of oncologic patients with COVID-19. Method: A systematic review of the literature was carried out in the PubMed database, utilizing the descriptors COVID-19, SARS-CoV-2, 2019-nCoV, n-CoV and coronavirus combined with cancer, tumor and malignancy. Results: Of the 821 articles found, nine texts were included in the qualitative analysis, totaling 39 individuals, predominantly male ( $74.4 \%$ men): China (6 texts, 35 patients), Nigeria (1 text, 1 patient), France (1 text, 2 patients), Denmark (1 text, 1 patient). Lung $(51.3 \% ; n=20)$ and breast $(10.3 \% ; n=4)$ cancers were the most prevalent. In $87.2 \%$ ( $n=34)$, information about life habits were not reported. Respiratory symptoms $(46.8 \% ; \mathrm{n}=18)$ and fever $(43.6 \% ; \mathrm{n}=17)$ stood out. Six patients died $(15.4 \%)$, four were discharged due to cure $(10.2 \%)$, two remained hospitalized until the study was published (5.2\%) and for the other patients $(69.3 \%, n=27)$, the manuscript did not inform the outcome . Conclusion: Adequate care for this special group of patients when infected with the new coronavirus is essential in pursuing better outcomes. Further studies are still needed to better understand how COVID-19 behaves in cancer patients. Key words: Coronavirus Infections; Neoplasms/epidemiology; Review.

\section{Resumen}

Introducción: Desde el comienzo de la pandemia de Covid-19, los estudios indican que los pacientes con cáncer representan uno de los principales grupos de riesgo. Objetivo: Sintetizar la literatura sobre el perfil clínicoepidemiológico de pacientes con cáncer además de Covid-19. Método: Revisión integral de la literatura utilizando la base de datos PubMed, con los descriptores COVID-19, SARS-CoV-2, 2019-nCoV, n-CoV y coronavirus combinados con cáncer, tumor y malignidad. Resultados: De los 821 artículos encontrados, se incluyeron nueve textos en el análisis cualitativo, totalizando 39 individuos, predominantemente hombres ( $74.4 \%$ hombres): China (6 textos, 35 pacientes), Nigeria (1 texto, 1 paciente), Francia (1 texto, 2 pacientes), Dinamarca (1 texto, 1 paciente). Los cánceres de pulmón $(51,3 \% ; n=20)$ y de mama $(10,3 \% ; n=4)$ fueron los más prevalentes. En $87,2 \%(\mathrm{n}=34)$ del total, no se informó ninguna información acerca de hábitos de vida. Se destacaron los síntomas respiratorios $(46,8 \% ; n=18)$ y fiebre $(43,6 \% ; n=17)$. Seis pacientes fallecieron $(15,4 \%)$, cuatro fueron dados de alta por cura $(10,2 \%)$, dos permanecieron hospitalizados hasta que se publicó el estudio $(5,2 \%)$ y en los otros $(69,3 \%, \mathrm{n}=27)$ el manuscrito no informó el resultado. Conclusión: La atención adecuada para este grupo especial de pacientes cuando se infectan con el nuevo coronavirus es esencial en la búsqueda de mejores resultados. Todavía se necesitan más estudios para comprender mejor cómo se comporta Covid-19 en pacientes con cáncer. Palabras clave: Infecciones por Coronavirus; Neoplasias/epidemiología; Revisión.

\footnotetext{
${ }^{1}$ Acadêmica de Medicina da Universidade Federal de Alagoas (UFAL). Campus Arapiraca. Arapiraca (AL), Brasil. Orcid iD: https://orcid.org/0000-0002-7956-4233

${ }^{2}$ Acadêmico de Medicina da UFAL. Campus Arapiraca. Arapiraca (AL), Brasil. Orcid iD: https://orcid.org/0000-0002-9934-4174

${ }^{3}$ Acadêmico de Medicina da UFAL. Campus Arapiraca. Arapiraca (AL), Brasil. Orcid iD: https://orcid.org/0000-0001-8567-7085

${ }^{4}$ Acadêmica de Medicina da UFAL. Campus Arapiraca. Arapiraca (AL), Brasil. Orcid iD: http://orcid.org/0000-0002-4186-6287

${ }^{5}$ Acadêmica de Medicina da UFAL. Campus Arapiraca. Arapiraca (AL), Brasil. Orcid iD: https://orcid.org/0000-0001-6920-937X

${ }^{6}$ Acadêmico de Medicina da UFAL. Campus Arapiraca. Arapiraca (AL), Brasil. Orcid iD: https://orcid.org/0000-0001-7144-1709

${ }^{7}$ Acadêmica de Medicina da UFAL. Campus Arapiraca. Arapiraca (AL), Brasil. Orcid iD: https://orcid.org/0000-0002-2119-3855

${ }^{8}$ Acadêmico de Medicina da UFAL. Campus Arapiraca. Arapiraca (AL), Brasil. Orcid iD: https://orcid.org/0000-0002-8467-8657

${ }^{9}$ Acadêmico de Medicina da UFAL. Campus Arapiraca. Arapiraca (AL), Brasil. Orcid iD: https://orcid.org/0000-0001-7458-1790

${ }^{10}$ Acadêmico de Medicina da UFAL. Campus Arapiraca. Arapiraca (AL), Brasil. Orcid iD: https://orcid.org/0000-0002-8212-4853

${ }^{11}$ Doutor em Saúde Pública/Epidemiologia pela Fundação Oswaldo Cruz (Fiocruz). Arapiraca (AL), Brasil. Orcid iD: http://orcid.org/0000-0003-0837-8254

Endereço para correspondência: Carlos Dornels Freire de Souza. Av. Manoel Severino Barbosa - Bom Sucesso (Complexo de Ciências Médicas - UFAL).

Arapiraca (AL), Brasil. CEP 57309-005. E-mail: carlos.freire@arapiraca.ufal.br
} 


\section{INTRODUÇÃO}

O novo coronavírus foi registrado pela primeira vez na cidade de Wuhan, capital da província de Hubei, na China ${ }^{1}$. Batizado de Sars-CoV-2 (severe acute respiratory syndrome coronavirus 2), o vírus, responsável pela Covid-19 (coronavirus disease 2019), rapidamente se espalhou pelo mundo, resultando em Emergência de Saúde Pública em fevereiro de 2020. No mês seguinte (11 de março), a Organização Mundial da Saúde declarou pandemia² .

Mesmo com os esforços que vêm sendo tomados para tentar conter os avanços da pandemia, em 14 de maio, já eram 4,3 milhóes de casos confirmados e $278 \mathrm{mil}$ mortes em decorrência do novo coronavírus no mundo. Estados Unidos (EUA) (1,3 milhôes) e Rússia (252 mil) ocupavam as duas primeiras posiçóes em número de casos confirmados, respectivamente. Em número de mortes, EUA (84 mil) e Reino Unido (33 mil) eram os primeiros colocados $^{3}$.

Entender o papel das doenças crônicas, a exemplo do câncer, como modificadoras e/ou agravantes do curso natural da Covid-19, ganha papel importante no cenário de pandemia. Pacientes com câncer correspondem a um grupo populacional vulnerável, pois apresentam maiores chances de apresentar a forma grave da doença em comparação com pacientes sem câncer ${ }^{4}$. A própria doença e seus tratamentos, como quimioterapia e radioterapia, são imunossupressores ${ }^{5,6}$, podendo elevar o risco de agravamento e morte pela Covid-19. Além disso, esses pacientes são frequentemente chamados ao hospital para tratamento e monitoramento, o que aumenta o risco de contrair Covid-194,6,7.

Considerando a importância desse grupo de risco e a ausência de investigaçôes a respeito, este trabalho buscou sintetizar a literatura no que se refere ao perfil clínico-epidemiológico de pacientes oncológicos com Covid-19.

\section{MÉTODO}

Revisão integrativa da literatura, incluindo artigos científicos que detalhassem as características clínicas e epidemiológicas dos pacientes oncológicos com Covid-19. Foram selecionados artigos publicados na base de dados PubMed entre os dias 1 de janeiro e 30 de abril de 2020, utilizando os descritores COVID-19, SARS-CoV-2, 2019- $n$ CoV, $n$-CoV e coronavirus combinados com cancer, tumor e malignancy. A coleta foi realizada em 30 de abril de 2020.

No estudo, foram incluídos ensaios clínicos, coortes, cross-sectional, casos clínicos e série de casos (publicados ou em preprint). Os seguintes critérios de exclusão foram adotados: boletins epidemiológicos governamentais, comentários, revisóes de literatura e artigos sem acesso ao conteúdo completo. Após a busca dos textos, três autores, de modo independente, executaram as etapas seguintes da investigação: 1- leitura do título e resumo; 2- leitura integral artigo; 3- coleta dos dados relativos à ocupação e montagem do banco de dados. A análise foi conduzida por dois outros pesquisadores de modo independente. Em seguida, as divergências foram analisadas pela equipe de investigação.

\section{RESULTADOS}

Inicialmente, 821 artigos foram encontrados. Destes, 92 foram selecionados para a leitura completa. Ao final, apenas nove textos ${ }^{4,7-14}$ possuíam informaçōes clínicas e epidemiológicas de pacientes com câncer que foram infectados pelo novo coronavírus, totalizando 39 indivíduos, com predomínio do sexo masculino (74,4\%), e de quatro países: China (6 textos, 35 pacientes); Nigéria (1 texto, 1 paciente); França (1 texto, 2 pacientes); e Dinamarca (1 texto, 1 paciente) (Figura 1 e Tabela 1 ).

Entre os tipos de cânceres, destacou-se o de pulmão, correspondendo a $51,3 \%(\mathrm{n}=20)$ dos pacientes, seguido do câncer de mama, com 10,3\% $(\mathrm{n}=4)$, e do câncer de cólon, com 7,7\% ( $\mathrm{n}=3)$. Os demais tipos, quando agrupados, somaram os $28,7 \%(\mathrm{n}=12)$ restantes. Sobre hábitos de vida, 12,8\% $(\mathrm{n}=5)$ dos indivíduos eram fumantes, sendo que, em $87,2 \%(n=34)$, esse dado não foi informado. Entre os sinais e sintomas apresentados, destacaram-se: sintomas respiratórios $(46,8 \% ; \mathrm{n}=18)$ e febre $(43,6 \% ; n=17)$. No que se refere ao desfecho, seis pacientes foram a óbito $(15,4 \%)$, quatro receberam alta por cura $(10,2 \%)$, dois permaneceram internados até a publicação do estudo $(5,2 \%)$ e os demais $(69,3 \%, n=27)$ não foram informados (Tabela 2).

\section{DISCUSSÃO}

O novo coronavírus tornou-se uma ameaça mundial e a principal preocupação sanitária do século XXI. Entre os grupos de risco, a literatura tem apontado que pacientes com comorbidades são mais suscetíveis a manifestar complicaçôes relacionadas ao novo coronavírus ${ }^{6,7}$.

De acordo com um estudo de coorte chinês, envolvendo 1.590 casos de Covid-19, dos quais 18 pacientes apresentavam câncer, observou-se que esses pacientes tiveram um risco maior de desenvolver eventos graves (admissão na unidade de terapia intensiva, necessidade de ventilaçáo invasiva ou morte) em comparação aos pacientes sem câncer: $39 \%$ versus $8 \%$, respectivamente ${ }^{4}$.

Diante do contexto global, a oncologia passou a ser afetada nas suas mais diversas formas. $\mathrm{Na}$ prática, o 


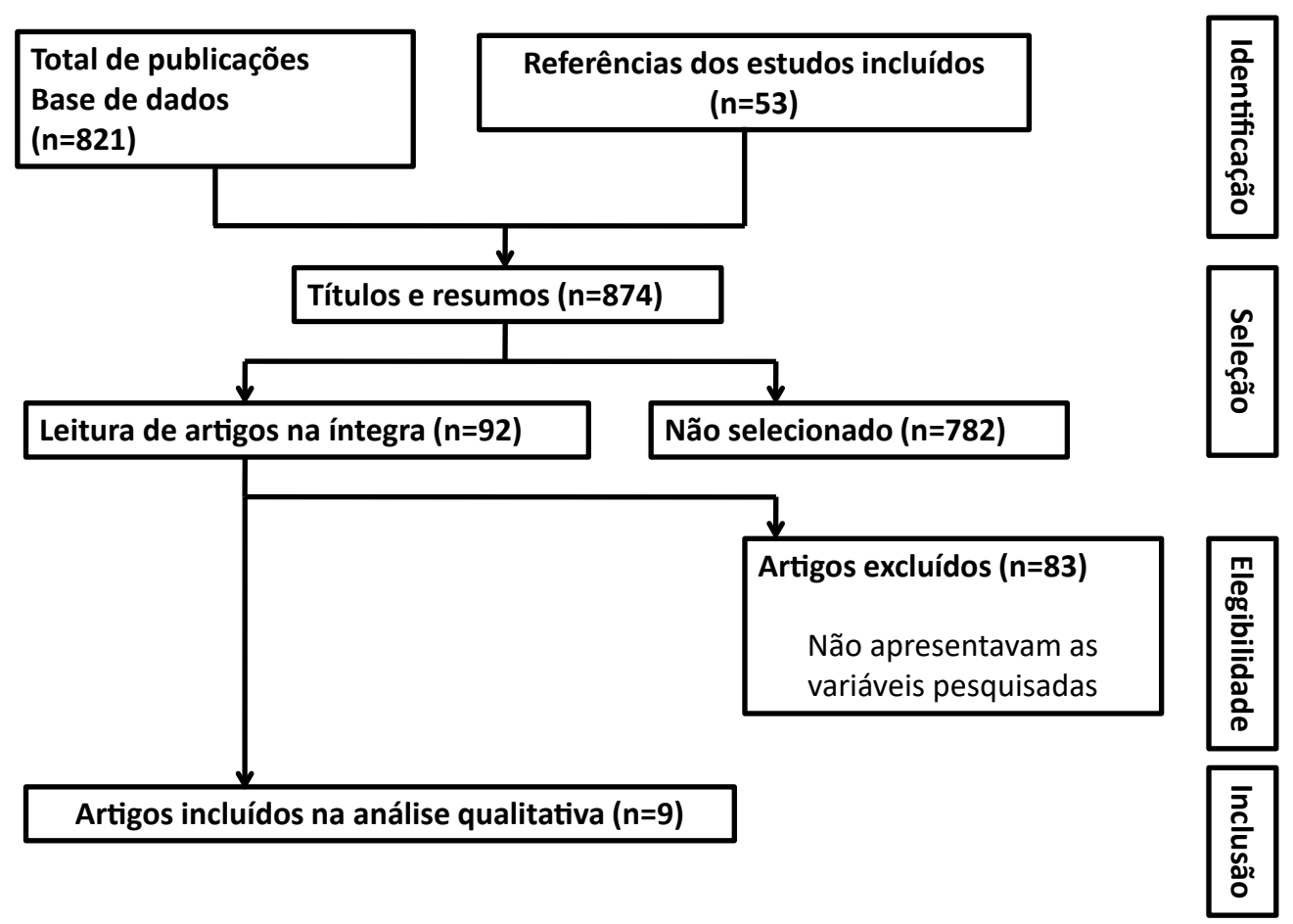

Figura 1. Fluxograma de seleção dos artigos, 2020

Tabela 1. Caracterização geral dos artigos incluídos no estudo, 2020

\begin{tabular}{|c|c|c|c|c|c|c|}
\hline Estudo & País & $\begin{array}{l}\text { N total } \\
\text { (câncer) }\end{array}$ & Tipo de estudo & Homens & Mulheres & Estrutura etária \\
\hline Liang et al. ${ }^{4}$ & China & 18 & Retrospectivo & 12 & 6 & Média: 63,1 anos \\
\hline Leonetti et al. ${ }^{7}$ & França & 2 & Relato de caso & 2 & 0 & Idade: 57 anos \\
\hline Tian et al. ${ }^{8}$ & China & 2 & Relato de caso & 1 & 1 & Média: 78,5 anos \\
\hline Qu et al. ${ }^{9}$ & China & 1 & Relato de caso & 0 & 1 & Idade: 46 anos \\
\hline Salako et al. ${ }^{10}$ & Nigéria & 1 & Transversal & 1 & 0 & Idade: 67 anos \\
\hline Yu et al. ${ }^{11}$ & China & 12 & Retrospectivo & 10 & 2 & $\begin{array}{c}\text { Média de idade: } 66 \\
\text { anos }\end{array}$ \\
\hline Zhang et al. ${ }^{12}$ & China & 1 & Relato de caso & 1 & 0 & Idade: 57 anos \\
\hline Ouyang et al. ${ }^{13}$ & China & 1 & Relato de caso & 1 & 0 & 57 anos \\
\hline Suppli et al. ${ }^{14}$ & Dinamarca & 1 & Relato de caso & 1 & 0 & 74 anos \\
\hline Total & & 39 & & $74,4 \%$ & $25,6 \%$ & Média de idade: 62,85 \\
\hline
\end{tabular}

tratamento do câncer, seja ele medicamentoso ou cirúrgico em paciente com Covid-19, é questionado pela falta de evidências e recomendaçóes sólidas quanto ao manejo desse grupo clínico. Além disso, pacientes oncológicos precisam de cuidados contínuos, e a manutenção do cuidado faz com que seja necessária a ida a ambientes hospitalares, aumentando a probabilidade de exposição ao vírus ${ }^{6}$.

A literatura científica aponta que os pacientes com câncer, em razão da sua estrutura etária (mais velhos), possuem outros fatores de risco (como hipertensão e diabetes) que, somados ao câncer, torna a Covid-19 muito mais agressiva. Além disso, tem sido notado que os pacientes que foram submetidos a tratamentos mais invasivos, como cirurgia e quimioterapia, apresentam um risco maior de evoluírem com eventos clínicos mais graves $^{4,10-15}$.

Pacientes oncológicos, por si só, já são mais desafiadores. Tanto a doença em si quanto o tratamento são capazes de promover modificaçóes fisiológicas que comprometem a imunidade e alteram o modo como o organismo se comporta frente a outras doenças, como as infecciosas, por exemplo ${ }^{11-13}$. Nesse contexto, o diagnóstico, a apresentação clínica e o suporte terapêutico tornam-se mais difíceis para a comunidade médica. 
Tabela 2. Caracterização clínica e epidemiológica (n=39), 2020

\begin{tabular}{|c|c|c|}
\hline Variável & \multirow{2}{*}{$\mathbf{N}$} & \multirow{2}{*}{$\%$} \\
\hline Tipo de câncer & & \\
\hline Pulmão & 20 & 51,3 \\
\hline Mama & 4 & 10,3 \\
\hline $\operatorname{Rim}$ & 1 & 2,6 \\
\hline Reto & 2 & 5,1 \\
\hline Cólon & 3 & 7,7 \\
\hline Colorretal & 1 & 2,6 \\
\hline Pâncreas & 1 & 2,6 \\
\hline Urotelial & 1 & 2,6 \\
\hline Bexiga & 2 & 5,1 \\
\hline Adrenal & 1 & 2,6 \\
\hline Tireoide & 1 & 2,6 \\
\hline Mieloma múltiplo & 1 & 2,6 \\
\hline Linfoma & 1 & 2,6 \\
\hline \multicolumn{3}{|l|}{ Sinais/sintomas } \\
\hline Assintomático & 1 & 2,6 \\
\hline Febre & 17 & 43,6 \\
\hline Tosse & 9 & 23,6 \\
\hline Dispneia & 4 & 10,3 \\
\hline Mialgia & 3 & 7,7 \\
\hline Fadiga & 2 & 5,2 \\
\hline Diarreia & 1 & 2,6 \\
\hline Náusea ou vômito & 1 & 2,6 \\
\hline Falta de ar & 4 & 10,3 \\
\hline Hipóxia & 1 & 2,6 \\
\hline Sudorese & 1 & 2,6 \\
\hline Anosmia & 1 & 2,6 \\
\hline Ageusia & 1 & 2,6 \\
\hline \multicolumn{3}{|l|}{ Hábitos de vida } \\
\hline Ex-fumante & 5 & $12,8 \%$ \\
\hline Não informado & 34 & $87,2 \%$ \\
\hline \multicolumn{3}{|l|}{ Imagem } \\
\hline Achados radiológicos não especificados & 10 & 25,6 \\
\hline Pneumonia bilateral & 3 & 7,7 \\
\hline Opacidade em vidro fosco & 5 & 12,8 \\
\hline Linfadenopatia & 1 & 2,6 \\
\hline Crazy paving & 1 & 2,6 \\
\hline Não informado & 19 & 48,7 \\
\hline \multicolumn{3}{|l|}{ Status da doença } \\
\hline Leve & 3 & 7,7 \\
\hline Moderado & 1 & 2,6 \\
\hline Severo/não grave & 3 & 7,7 \\
\hline Grave (crítico) & 2 & 5,1 \\
\hline Não informado & 30 & 76,9 \\
\hline
\end{tabular}

Tabela 2. continuação

\begin{tabular}{|c|c|c|}
\hline Variável & \multirow{2}{*}{$\mathbf{N}$} & \multirow{2}{*}{$\%$} \\
\hline Tratamento & & \\
\hline Antibiótico & 3 & 7,7 \\
\hline Antiviral & 1 & 2,6 \\
\hline Oxigenoterapia & 2 & 5,1 \\
\hline Ventilação mecânica & 1 & 2,6 \\
\hline Medicina tradicional chinesa & 1 & 2,6 \\
\hline Não informado & 31 & 79,5 \\
\hline \multicolumn{3}{|l|}{ Desfecho } \\
\hline Óbito & 6 & 15,4 \\
\hline Cura/alta & 4 & 10,2 \\
\hline Permanece internado & 2 & 5,1 \\
\hline Não informado & 27 & 69,3 \\
\hline
\end{tabular}

Exemplo disso são os achados radiográficos, nos quais, muitas vezes, características típicas de Covid-19 podem não significar a presença da doença, uma vez que já há relatos na literatura que mostraram a incompatibilidade entre exames de imagem e testes de reaçáo em cadeia de polimerase (PCR) que deram negativos ${ }^{6}$. Ademais, a literatura evidenciou que pode haver semelhanças entre as alteraçóes radiológicas causadas pela Covid-19 e aquelas motivadas pelo próprio câncer, em especial o de pulmão ${ }^{8,9}$.

O mesmo pode acontecer com os demais sinais/ sintomas, sobretudo com aqueles ligados ao sistema respiratório e febre. Em indivíduos com câncer de pulmão, por exemplo, queixas clínicas relacionadas com esse sistema são predominantes. Estabelecer a relação entre as queixas apresentadas e a causa provável representa um desafio adicional para os médicos. Esse aspecto demonstra a necessidade de diretrizes clínicas que possam nortear os profissionais no manejo desses pacientes.

Além dos efeitos prejudiciais da pandemia para os pacientes oncológicos, é necessário destacar que, na tentativa de conter a disseminação do vírus, as atividades clínicas foram reduzidas. Essa conduta, juntamente com o isolamento social, pode trazer como consequência, em longo prazo, um menor índice de diagnóstico de cânceres em estágios mais iniciais. No futuro, o diagnóstico tardio pode impactar na sobrevivência desses novos diagnósticos ${ }^{10}$. Segundo uma análise realizada na Inglaterra, está previsto um acréscimo de 6.270 mortes (incremento de 20\%) nos próximos 12 meses em pacientes com novos diagnósticos de câncer como consequência da pandemia ${ }^{15}$.

O reduzido número de investigaçóes sobre a temática e a pequena população estudada são limitaçôes deste estudo. Essa carência impede a definição de um perfil clínico dos pacientes oncológicos infectados pelo Sars-CoV-2 e a identificação de subgrupos de maior risco. 


\section{CONCLUSÃO}

O cuidado adequado a esse grupo especial de pacientes quando infectados pelo novo coronavírus é fundamental para busca de melhores desfechos. Neste estudo, o perfil foi caracterizado pelo predomínio do sexo masculino, câncer de pulmáo e sintomas respiratórios. Observou-se ainda alta letalidade.

Muitos esforços estão sendo empreendidos para melhor entender as particularidades dos pacientes com câncer infectados pelo novo coronavírus. A escassez de investigaçôes sobre os efeitos do vírus nos indivíduos com câncer e a pequena populaçáo incluída nos estudos demonstram a necessidade urgente de novas investigaçôes científicas direcionadas para esse público.

\section{CONTRIBUIÇÕES}

Todos os autores contribuíram substancialmente na concepção e no planejamento do estudo; na obtenção, análise e interpretaçáo dos dados; na redação e revisão crítica; e aprovaram a versão final a ser publicada.

\section{DECLARAÇÃO DE CONFLITO DE INTERESSES}

Nada a declarar.

\section{FONTES DE FINANCIAMENTO}

Não há.

\section{REFERÊNCIAS}

1. Hui DS, Azhar EI, Madani TA, et al. The continuing 2019-nCoV epidemic threat of novel coronaviruses to global health - The latest 2019 novel coronavirus outbreak in Wuhan, China. Int J Infect Dis. 2020;91:264-66. doi: https://doi.org/10.1016/j.ijid.2020.01.009

2. Organização Pan-Americana da Saúde (BR). Brasília, DF: OPAS; [data desconhecida]. Folha informativa COVID-19 (doença causada pelo novo coronavírus); 2020 maio 25 [acesso 2020 maio 14]. Disponível em: https://www.paho.org/bra/index.php?option=com_con tent $\&$ view $=$ article $\&$ id $=6101$ : covid $19 \&$ Itemid $=875$

3. World Health Organization. Coronavirus disease (COVID-19). Situation Report; 114. Geneva: WHO; 2020 May 13 [cited 2020 May 14]. Available from: https://www.who.int/docs/default-source/coronaviruse/ situation-reports/20200513-covid-19-sitrep-114. pdf?sfvrsn=17ebbbe_4

4. Liang W, Guan W, Chen R, et al. Cancer patients in SARS-CoV-2 infection: a nationwide analysis in China.
Lancet Oncol. 2020;21(3):335-7. doi: https://doi. org/10.1016/S1470-2045(20)30096-6

5. Sica A, Massarotti M. Myeloid suppressor cells in cancer and autoimmunity. J Autoimmun. 2017;85:117-25. doi: https://doi.org/10.1016/j.jaut.2017.07.010

6. Moujaess E, Kourie HR, Ghosn M. Cancer patients and research during COVID-19 pandemic: a systematic review of current evidence. Crit Rev Oncol Hematol. 2020 Apr 22;150:102972. doi: https://doi.org/10.1016/j. critrevonc.2020.102972

7. Leonetti A, Facchinetti F, Zielli T, et al. COVID-19 in lung cancer patients receiving ALK/ROS1 inhibitors. Eur J Cancer. 2020 Jun;132:122-4. doi: https://doi. org/10.1016/j.ejca.2020.04.004

8. Tian S, Hu W, Niu L, et al. Pulmonary pathology of earlyphase 2019 novel coronavirus (covid-19) pneumonia in two patients with lung cancer. J Thorac Oncol. 2020;15(5):700-4. doi: https://doi.org/10.1016/j. jtho.2020.02.010

9. $\mathrm{Qu} \mathrm{J}$, Yang R, Song L, et al. Atypical lung feature on chest $\mathrm{CT}$ in a lung adenocarcinoma cancer patient infected with COVID-19. Ann Oncol. 2020 Mar 9. doi: https:// doi.org/10.1016/j.annonc.2020.03.001 Epub ahead of print. PubMed PMID: 32165205.

10. Salako O, Okunade K, Allsop M, et al. Upheaval in cancer care during the COVID-19 outbreak. Ecancermedicalscience. 2020 Apr 1;14:ed97. doi: https:// doi.org/10.3332/ecancer.2020.ed97

11. Yu J, Ouyang W, Chua MLK, et al. SARS-CoV-2 Transmission in patients with cancer at a tertiary care hospital in Wuhan, China. JAMA Oncol. 2020 Mar 25;e200980. doi: https://doi.org/10.1001/ jamaoncol.2020.0980

12. Zhang H, Xie C, Huang Y. Treatment and outcome of a patient with lung cancer infected with severe acute respiratory syndrome Coronavirus-2. J Thorac Oncol. 2020 May;15(5):e63-e64. doi: https://doi.org/10.1016/j. jtho.2020.02.025

13. Ouyang W, Yu J, Zhang J, et al. Alert to potential contagiousness: a case of lung cancer with asymptomatic SARS-CoV-2 infection. J Thorac Oncol. 2020 Apr;S1556-0864(20)30300-2. doi: https://doi. org/10.1016/j.jtho.2020.04.005 Epub ahead of print. PubMed PMID: 32305592.

14. Suppli MH, Blanck SR, Elgaard T, et al. Early appearance of coronavirus disease 2019 associated pulmonary infiltrates during daily radiotherapy imaging for lung cancer. J Thorac Oncol. 2020 Apr;S1556-0864(20)30299-9. doi: https://doi. org/10.1016/j.jtho.2020.04.004 Epub ahead of print. PubMed PMID: 32283316. 
15. Banerjee A, Pasea L, Harris S, et al. Estimating excess 1-year mortality from COVID-19 according to underlying conditions and age in England: a rapid analysis using NHS health records in 3.8 million adults. MedRxiv [Preprint]. 2020 Mar 24. doi: https://doi. org/10.1101/2020.03.22.20040287 